\title{
The Normal-Laplace Distribution and its Relatives.
}

\author{
William J. Reed* \\ Department of Mathematics and Statistics, \\ University of Victoria, \\ PO Box 3045, Victoria, B.C., \\ Canada V8W 3P4 \\ (e-mail:reed@math.uvic.ca).
}

October 21, 2004

\begin{abstract}
The normal-Laplace (NL) distribution results from convolving independent normally distributed and Laplace distributed components. It is the distribution of the stopped state of a Brownian motion with normally distributed starting value if the stopping hazard rate is constant. Properties of the NL distribution discussed in the article include its shape and tail behaviour (fatter than the normal), its moments and its infinitely divisibility. The double Pareto-lognormal distribution is that of an exponentiated normal-Laplace random variable and provides a useful parametric form for modelling size distributions. The generalized normal-Laplace (GNL) distribution is both infinitely divisible and closed under summation. It is possible to construct a Lévy process whose increments follow the GNL distribution. Such a Lévy motion can be used to model the movement of the logarithmic price of a financial asset. An option pricing formula is derived for such an asset.
\end{abstract}

Keywords: fat tails; generalized normal-Laplace distribution; double Pareto-lognormal distribution; Brownian-Laplace motion; Lévy process; financial returns; option value.

*Research supported by NSERC grant OGP 7252. 


\section{Introduction.}

Although the normal (Gaussian) distribution plays a central role in basic statistics, it has long been recognized that the empirical distributions of many phenomena modelled by the normal distribution sometimes do not follow closely to the Gausssian shape. For example E. B. Wilson (1923) in a paper in the Journal of the American Statistical Assciation stated that "the frequency we actually meet in everyday work in economics, biometrics, or vital statistics often fails to conform closely to the so-called normal distribution". In recent years the huge burst of research interest in financial modelling along with the availability of high frequency price data and the concomitant realisation that logarithmic price returns do not follow exactly a normal distribution (see e.g Rydberg, 2000), as previously assumed, has led to a search for more realistic alternative parametric models.

Distributions can of course differ from one another in myriad ways, but those for which empirical distributions modelled by the normal tend to differ from the normal can be broadly classified into two kinds viz. the presence of skewness; and the fact of having fatter tails than the normal (leptokurtosis).

A number of alternative parametric forms have been used to deal with the presence of leptokurtososis, ranging from the Student- $t$ (including the $t_{(1)}$ or Cauchy) distribution to the logistic and Laplace distributions. The Laplace distribution can be extended to an asymmetric form (skew-Laplace) as well as to the generalized Laplace distribution (Kotz et al., 2001). Other distributions of this type, which are parameter rich and can incorporate both skewness and 
kurtososis are the generalized hyperbolic distribution (Barndorff Nielsen, 1977;

Eberlein and Keller, 1995) and its subclass the normal inverse Gaussian distribution (Barndorff Nielsen, 1997). These latter distributions have all been used recently in finance to model logarithmic price returns.

It is the purpose of this paper to present a new distribution which (in its symmetric form) behaves somewhat like the normal distribution in the middle of its range, and like the Laplace distribution in its tails. This distribution, named herin as the normal-Laplace distribution results from convolving independent normal and Laplace components. Skewness can be introduced into the distribution by using a skew-Laplace component in the convolution.

In Sec. 2 the distribution is defined and its genesis and properties are discussed. In Sec. 3 the double Pareto-lognormal distribution (which is that of an exponentiated normal-Laplace random variable) is briefly discussed along with its use in modelling the size distribution of various phenomena. Also in Sec. 3 the generalized normal-Laplace distribution is introduced and some of its properties discussed. In Sec. 4 the construction of a Lévy process (termed BrownianLaplace motion) whose increments follow the generalized normal-Laplace distribution is described along with its potenetial use in financial modelling. This includes the determination of the option value of a European call option for an asset whose logarithmic price follows Brownian-Laplace motion. In Sec. 5 parameter estimation for the normal-Laplace and generalized normal-Laplace distributions is discussed. 


\section{The Normal-Laplace distribution.}

\section{Definition.}

The basic normal-Laplace distribution can be defined in terms of its cumulative distribution function (cdf) which for all real $y$ is

$F(y)=\Phi\left(\frac{y-\mu}{\sigma}\right)-\phi\left(\frac{y-\mu}{\sigma}\right) \frac{\beta R(\alpha \sigma-(y-\mu) / \sigma)-\alpha R(\beta \sigma+(y-\mu) / \sigma)}{\alpha+\beta}$

where $\Phi$ and $\phi$ are the cdf and probability density function (pdf) of a standard normal random variable and $R$ is Mills' ratio :

$$
R(z)=\frac{\Phi^{c}(z)}{\phi(z)}=\frac{1-\Phi(z)}{\phi(z)}
$$

The location parameter $\mu$ can assume any real value while the scale parameter $\sigma$ and the other two parameters $\alpha$ and $\beta$, which determine tail behaviour, are assumed to be positive.

The corresponding density (pdf)is

$$
f(y)=\frac{\alpha \beta}{\alpha+\beta} \phi\left(\frac{y-\mu}{\sigma}\right)[R(\alpha \sigma-(y-\mu) / \sigma)+R(\beta \sigma+(y-\mu) / \sigma)] .
$$

We shall write

$$
Y \sim \mathrm{NL}\left(\mu, \sigma^{2}, \alpha, \beta\right)
$$

to indicate that a random variable $Y$ has such a distribution.

\section{Genesis.}

The distribution arises as the convolution of a normal distribution and an asymmetric Laplace i.e $Y \sim \mathrm{NL}\left(\mu, \sigma^{2}, \alpha, \beta\right)$ can be represented as

$$
Y \stackrel{d}{=} Z+W
$$


where $Z$ and $W$ are independent random variables with $Z \sim \mathrm{N}\left(\mu, \sigma^{2}\right)$ and $W$ following an asymmetric Laplace distribution with pdf

$$
f_{W}(w)= \begin{cases}\frac{\alpha \beta}{\alpha+\beta} e^{\beta w}, & \text { for } w \leq 0 \\ \frac{\alpha \beta}{\alpha+\beta} e^{-\alpha w}, & \text { for } w>0\end{cases}
$$

Such a convolution might naturally occur if a Brownian motion

$$
d X=\nu d t+\tau d w
$$

with initial state $X_{0} \sim \mathrm{N}\left(\mu, \sigma^{2}\right)$ were to be observed at an exponentially distributed time $T$; or put another way if such a Brownian motion were stopped (or "killed", or observed) with a constant hazard rate $\lambda$, and the stopped state $X(T)$ observed. This follows from the fact that the state of the Brownian motion (6) with fixed (non-random) initial state after an exponentially distributed time follows an asymmetric Laplace distribution (e.g. Kotz et al., 2001, p. 145).

Thus for example, if the logarithmic price of a stock or other financial asset $\left\{\log P_{t}\right\}_{t \geq 0}$ followed Brownian motion, as has been widely assumed, the $\log$ (price) at the time of the first trade on a fixed day $n$, say, could be expected to follow a distribution close to a normal-Laplace. This is because the $\log$ (price) at the start of day $n$ would be normally distributed, while under the assumption that trades on day $n$ occur in a Poisson process, the time until the first trade would be exponentially distributed.

\section{Some properties.}

Since a Laplace random variable can be represented as the difference between two exponentially distributed variates (Kotz et al., 2001) it follows from (4) that 
an $\mathrm{NL}\left(\mu, \sigma^{2}, \alpha, \beta\right)$ random variable can be expressed as

$$
Y \stackrel{d}{=} \mu+\sigma Z+E_{1} / \alpha-E_{2} / \beta
$$

where $E_{1}, E_{2}$ are independent standard exponential deviates and $Z$ is a standard normal deviate independent of $E_{1}$ and $E_{2}$. This provides a conveneient way to simulate pseudo-random numbers from the NL distribution.

Kotz et al., (2001, p. 149) provide several other representations of asymmetric Laplace random variables. With suitable adjustment (addition of a $\mathrm{N}\left(\mu, \sigma^{2}\right)$ component) these all carry over for normal-Laplace random variables. Some other properties are:

- Shape and tail behaviour. The normal-Laplace pdf is smooth (differentiable) and has a single mode. It decays to zero as $y \rightarrow \pm \infty$. In the case $\alpha=\beta$ it is symmetric and bell-shaped, occupying a intermediate position between a normal and a Laplace distribution. Fig. 1 shows the $\operatorname{NL}(0,1 / 3,1 / \sqrt{3}, 1 / \sqrt{3})$ distribution (solid curve), which has mean zero and variance 1 along with the normal (dot-dash) and Laplace (dashed) distributions with the same mean and variance. Tha parameters $\alpha$ and $\beta$ determine the behaviour in the left and right tails repectively. A small values of either of these parameters corresponds to heavyiness in the corresponding tail. Fig. 2 shows the $\mathrm{NL}(0,1,1, \beta)$ pdf for values of $\beta=1,1 / 2,1 / 3,1 / 4$ and $1 / 5$; while Fig. 3 shows the symmetric $\mathrm{NL}(0,1, \alpha, \alpha)$ pdf for values of $\alpha=2,1,3 / 4$ and $1 / 2$.

In comparison with the $\mathrm{N}\left(\mu, \sigma^{2}\right)$ distribution, the $\mathrm{NL}\left(\mu, \sigma^{2}, \alpha, \beta\right)$ distribution will always have more weight in the tails, in the sense that for $y$ suitably small $F(y)>\Phi((y-\mu) / \sigma)$, while for $y$ suitably large $1-F(y)>1-\Phi((y-\mu) / \sigma)$. 
This follows from the expression (1) for the cdf, since the term $\beta R(\alpha \sigma-(y-$ $\mu) / \sigma)-\alpha R(\beta \sigma+(y-\mu) / \sigma)$ is decreasing in $y$ from $\infty$ to $-\infty$ over the interval $(-\infty, \infty)$

If the NL distribution is thought of as a convolution of normal and Laplace components, it is the Laplace component which dominates in the tails in the sense that the tails decay exponentially i.e.

$$
f(y) \sim k_{1} e^{-\alpha y} \quad(y \rightarrow \infty) ; \quad f(y) \sim k_{2} e^{\beta y} \quad(y \rightarrow-\infty)
$$

where $k_{1}=\alpha \exp \left[\alpha \sigma+\alpha^{2} \sigma^{2} / 2\right]$ and $k_{2}=\beta \exp \left[-\beta \sigma+\beta^{2} \sigma^{2} / 2\right]$.

- Moment generating function (mgf). From the representation (4) it follows that the mgf of $N L\left(\alpha, \beta, \mu, \sigma^{2}\right)$ is the product of the mgfs of its normal and Laplace components. Precisely it is

$$
M_{Y}(s)=\frac{\alpha \beta \exp \left(\mu s+\sigma^{2} s^{2} / 2\right)}{(\alpha-s)(\beta+s)} .
$$

- Mean, variance and cumulants. Expanding the cumulant generating function, $K_{Y}(s)=\log M_{Y}(s)$, yields

$$
E(Y)=\mu+1 / \alpha-1 / \beta ; \quad \operatorname{var}(Y)=\sigma^{2}+1 / \alpha^{2}+1 / \beta^{2}
$$

Higher order cumulants are

$$
\kappa_{r}=(r-1) !\left(\alpha^{-r}+(-\beta)^{-r}\right), \quad \text { for integer } r>2 .
$$

In particular

$$
\kappa_{3}=2 / \alpha^{3}-2 / \beta^{3} ; \quad \kappa_{4}=6 / \alpha^{4}+6 / \beta^{4} .
$$

- Closure under linear transformation. The NL distribution is closed under 
linear transformation. Precisely if $Y \sim N L\left(\alpha, \beta, \mu, \sigma^{2}\right)$ and $a$ and $b$ are any constants, then $a Y+b \sim N L\left(\alpha / a, \beta / a, a \mu+b, a^{2} \sigma^{2}\right)$.

- Infinite divisibility. The NL distribution is infinitely divisible. This follows from writing its mgf as

$$
M_{Y}(s)=\left[\exp \left(\frac{\mu}{n} s+\frac{\sigma^{2}}{2 n} s^{2}\right)\left(\frac{\alpha}{\alpha-s}\right)^{1 / n}\left(\frac{\beta}{\beta+s}\right)^{1 / n}\right]^{n}
$$

for any integer $n>0$ and noting that the term in square brackets is the mgf of a random variable formed as $Z+G_{1}-G_{2}$, where $Z, G_{1}$ and $G_{2}$ are independent and $Z \sim N\left(\frac{\mu}{n}, \frac{\sigma^{2}}{n}\right)$ and $G_{1}$ and $G_{2}$ have gamma distributions with parameters $1 / n$ and $\alpha$ and $1 / n$ and $\beta$ respectively.

\section{Some special cases.}

From the representation (4) of the NL as a convolution of normal and Laplace components it is clear that as $\sigma \rightarrow 0$, the distribution tends to an asymmetric Laplace distribution; and as $\alpha, \beta \rightarrow \infty$, it tends to a normal distribution. If only $\beta=\infty$ the distribution is that of the sum of independent normal and exponential components and has a fatter tail than the normal only in the upper tail. In this case the pdf is

$$
f_{1}(y)=\alpha \phi\left(\frac{y-\mu}{\sigma}\right) R(\alpha \sigma-(y-\mu) / \sigma) .
$$

Similarly if only $\alpha=\infty$ the distribution exhibits extra-normal variation only in the lower tail and the pdf is

$$
f_{2}(y)=\beta \phi\left(\frac{y-\mu}{\sigma}\right) R(\beta \sigma+(y-\mu) / \sigma) .
$$


Clearly the general $\mathrm{NL}\left(\mu, \sigma^{2}, \alpha, \beta\right)$ pdf (2) can be represented as a mixture of the above pdfs as

$$
f(y)=\frac{\beta}{\alpha+\beta} f_{1}(y)+\frac{\alpha}{\alpha+\beta} f_{2}(y)
$$

A special case of some importance already mentioned (Fig. 3) is the symmetric normal-Laplace distribution arising when $\alpha=\beta$, with pdf

$$
f(y)=\frac{\alpha}{2} \phi\left(\frac{y-\mu}{\sigma}\right)[R(\alpha \sigma-(y-\mu) / \sigma)+R(\alpha \sigma+(y-\mu) / \sigma)] .
$$

\section{Related distributions.}

\subsection{The double Pareto-lognormal distribution.}

The double Pareto-lognormal distribution is related to the normal-Laplace distribution in the same way as the lognormal is related to the normal, i.e. a random variable $X$ for which $\log X \sim \mathrm{NL}\left(\mu, \sigma^{2}, \alpha, \beta\right)$ is defined as following the double Pareto-lognormal distribution. As such it could be termed the "log normal-Laplace". However the name 'double Pareto-lognormal' (which was coined because the distribution results from the product of double Pareto and lognormal components) has already been used (Reed \& Jorgensen, 2004). The double Pareto-lognormal (or $d P l N$ ) distribution shares many characteristics with the log-hyperbolic distribution (Barndorff-Nielsen, 1977). For example it exhibits power-law behaviour in both tails and has an approximately hyperbolic shape when the pdf is plotted logarithmic axes. Like the log-hyperbolic distribution, the dPIN distribution has proved useful in modelling size distributions. It has been shown to provide a very good fit to a variety of empirical size dis- 
tribution data (e.g. incomes and wealth, city sizes, particle sizes, oil field sizes etc. - see Reed \& Jorgensen, 2004).

\subsection{The generalized normal-Laplace distribution.}

While the NL distribution is infinitely divisible, it is not closed under the convolution operation i.e. sums of independent NL random variables do not themselves follow NL distributions. The generalized normal-Laplace is an extension of the NL distribution for which a closure property of this type holds. The advantage of this is that for such a class of distributions one can construct a Lévy motion for which the increments follow the given distribution. This is useful in financial applications for obtaining an alternative stochastic process model to Brownian motion for logarithmic prices, in which the increments (logarithmic returns) exhibit fatter tails than the normal distribution (something that has been widely observed in high frequency finance data).

The generalized-normal Laplace (GNL) distribution is defined as that of a random variable $X$ with characteristic function

$$
\phi_{G N L}(s)=\left[\frac{\alpha \beta \exp \left(i \mu s-\sigma^{2} s^{2} / 2\right)}{(\alpha-i s)(\beta+i s)}\right]^{\rho}
$$

and hence moment generating function

$$
M_{G N L}(s)=\left[\frac{\alpha \beta \exp \left(\mu s+\sigma^{2} s^{2} / 2\right)}{(\alpha-s)(\beta+s)}\right]^{\rho}
$$

where $\alpha, \beta, \rho$ and $\sigma$ are positive parameters, $-\infty<\mu<\infty$. Let

$$
X \sim \operatorname{GNL}\left(\mu, \sigma^{2}, \alpha, \beta, \rho\right)
$$


denote that the random variable $X$ follows such a distribution ${ }^{1}$. Writing the mgf as

$$
\exp \left(\rho \mu s+\rho \sigma^{2} s^{2} / 2\right)\left[\frac{\alpha}{\alpha-s}\right]^{\rho}\left[\frac{\beta}{\beta+s}\right]^{\rho}
$$

it can be seen that $X$ can be represented as

$$
X \stackrel{d}{=} \rho \mu+\sigma \sqrt{\rho} Z+\frac{1}{\alpha} G_{1}-\frac{1}{\beta} G_{2}
$$

where $Z, G_{1}$ and $G_{2}$ are independent with $Z \sim \mathrm{N}(0,1)$ and $G_{1}, G_{2}$ are gamma random variables with scale parameter 1 and shape parameter $\rho$, i.e. with probability density function (pdf)

$$
\gamma(u)=\frac{1}{\Gamma(\rho)} u^{\rho-1} e^{-u}
$$

From (16) it is easily established that the GNL is infinitely divisible. Furthermore sums of independent, identically distributed (iid) GNL random variables, with common $\alpha$ and $\beta$ parameters, also follow a GNL distribution.

The mean and variance of the $\operatorname{GNL}\left(\mu, \sigma^{2}, \alpha, \beta, \rho\right)$ distribution are

$$
\mathrm{E}(Y)=\rho\left(\mu+\frac{1}{\alpha}-\frac{1}{\beta}\right) ; \quad \operatorname{var}(Y)=\rho\left(\sigma^{2}+\frac{1}{\alpha^{2}}+\frac{1}{\beta^{2}}\right)
$$

while the higher order cumulants are (for $r>2$ )

$$
\kappa_{r}=\rho(r-1) !\left(\frac{1}{\alpha^{r}}+(-1)^{r} \frac{1}{\beta^{r}}\right)
$$

Note that the coefficient of kurtosis

$$
\kappa_{4} / \kappa_{2}^{2}=\frac{1}{\rho} \frac{3 !\left(\alpha^{4}+\beta^{4}\right)}{\left(\sigma^{2} \alpha^{2} \beta^{2}+\alpha^{2}+\beta^{2}\right)^{2}}
$$

\footnotetext{
${ }^{1}$ The distribution with the above mgf with $\mu=\sigma^{2}=0$ has been called the generalized Laplace distribution by Kotz et al., (2001) (it has also been called the Bessel function distribution and the variance-gamma distribution by other authors). The generalized normal-Laplace distribution defined above bears the same relation to the normal-Laplace distribution as does the generalized Laplace to the Laplace.
} 
is decreasing in $\rho$.

The parameters $\mu$ and $\sigma^{2}$ influence the central location and spread of the distribution; while $\alpha, \beta$ and $\rho$ affect the tail behaviour. Ceteris paribus decreasing $\alpha$ (or $\beta$ ) puts more weight into the upper (or lower) tail. When $\alpha=\beta$ the distribution is symmetric and in the limiting case $\alpha=\beta=\infty$ the GNL reduces to a normal distribution. Also increasing $\rho$ moves the shape of the distribution towards normality. In the case $\rho=1$ the GNL becomes an ordinary normalLaplace (NL) distribution. For finite values of $\alpha$ and $\beta$ the GNL distribution, like the NL distribution, has fatter tails than a normal distribution.

\section{A Lévy motion based on the GNL distribu- tion.}

We now consider a Lévy process $\left\{X_{t}\right\}_{t \geq 0}$, say for which the increments $X_{t+\tau}-$ $X_{\tau}$ have characteristic function $\left(\phi_{G N L}(s)\right)^{t}$ where $\phi_{G N L}$ is the characteristic function of the $\operatorname{GNL}\left(\mu, \sigma^{2}, \alpha, \beta, \rho\right)$ defined in (16) (such a construction is always possible for an infinitely divisible distribution - see e.g. Schoutens, 2003). It is not difficult to show that the Lévy triplet for this process is $\left(\rho \mu, \rho \sigma^{2}, \Lambda\right)$ where $\Lambda$ is the Lévy measure of asymmetric Laplace motion (see Kotz et al., 2001, p. 198). Laplace motion has an infinite number of jumps in any finite time interval (a pure jump process). The extension considered here adds a continuous Brownian component to Laplace motion. We shall thus call the process $\left\{X_{t}\right\}_{t \geq 0}$ defined above, Brownian-Laplace motion.

The increments $X_{t+\tau}-X_{\tau}$ of this process will follow a $\operatorname{GNL}\left(\mu, \sigma^{2}, \alpha, \beta, \rho t\right)$ 
distribution and will have fatter tails than the normal. However as $t$ increases the kurtosis of the distribution drops. Exactly this sort of behaviour has been observed in various studies on high-frequency financial data (e.g. Rydberg, 2000) - very little kurtosis in the distribution of logarithmic returns over long intervals but increasingly fat tails as the reporting interval is shortened. Thus Brownian-Laplace motion seems to provide a good model for the movement of logarithmic prices.

\subsection{Option pricing for assets with logarithmic prices fol- lowing Brownian-Laplace motion.}

We consider an asset whose price $S_{t}$ is given by

$$
S_{t}=S_{0} \exp \left(X_{t}\right)
$$

where $\left\{X_{t}\right\}_{t \geq 0}$ is a Brownian-Laplace motion with $X_{0}=0$ and parameters $\mu, \sigma^{2}, \alpha, \beta, \rho$. We wish to determine the risk-neutral valuation of a European call option on the asset with strike price $K$ at time $T$ and a discount rate $r$.

It can be shown using the Esscher equivalent martingale measure (see e.g. Schoutens, 2003, p. 77) that the option value can be expressed in a form similar to that of the Black-Scholes formula. Precisely

$$
O V=S_{0} \int_{\gamma}^{\infty} d_{G N L}^{* T}(x ; \theta+1) d x-e^{-r T} K \int_{\gamma}^{\infty} d_{G N L}^{* T}(x ; \theta) d x
$$

where $\gamma=\log \left(K / S_{0}\right)$ and

$$
d_{G N L}^{* T}(x ; \theta)=\frac{e^{\theta x} d_{G N L}^{* T}(x)}{\int_{-\infty}^{\infty} e^{\theta y} d_{G N L}^{* T}(y) d y}
$$

is the pdf of $X_{T}$ under the risk-neutral measure. Here $d_{G N L}^{* T}$ is the pdf of the $T$-fold convolution of the generalized normal-Laplace, $\operatorname{GNL}\left(\mu, \sigma^{2}, \alpha, \beta, \rho\right)$, 
distribution and $\theta$ is the unique solution to the following equation involving its $\operatorname{mgf}$

$$
\log M_{G N L}(\theta+1)-\log M_{G N L}(\theta)=r .
$$

The $T$-fold convolution of $\operatorname{GNL}\left(\mu, \sigma^{2}, \alpha, \beta, \rho\right)$ is $\operatorname{GNL}\left(\mu, \sigma^{2}, \alpha, \beta, \rho T\right)$ and so its moment generating function is (17) with $\rho$ replaced by $\rho T$. This provides the denominator of the expression (21) for the risk-neutral pdf.

Now let

$$
I_{\theta}=\int_{\gamma}^{\infty} d_{G N L}^{* T}(x ; \theta) d x=\frac{1}{\left[M_{G N L}(\theta)\right]^{T}} \int_{\gamma}^{\infty} e^{\theta x} d_{G N L}^{* T}(x)
$$

so that

$$
O V=I_{\theta+1}-I_{\theta} .
$$

Thus to evaluate the option value we need only evaluate the integral in (23). This can be done using the representation (18) of a GNL random variable as the sum of normal and positive and negative gamma components. Precisely the integral can be written

$$
\int_{0}^{\infty} g(u ; \alpha) \int_{0}^{\infty} g(v ; \beta) \int_{\gamma}^{\infty} e^{\theta x} \frac{1}{\sigma \sqrt{\rho T}} \phi\left(\frac{x-u+v-\mu \rho T}{\sigma \sqrt{\rho T}}\right) d x d v d u
$$

where

$$
g(x ; a)=\frac{a^{\rho T}}{\Gamma(\rho T)} x^{\rho T-1} e^{-a x}
$$

is the pdf of a gamma random variable with scale parameter $a$ and shape parameter $\rho T$; and $\phi$ is the pdf of a standard normal deviate. After completing the square in $x$ and evaluating the $x$ integral in terms of $\Phi^{c}$, the complementary cdf 
of a standard normal, the integral can be expressed

$$
\int_{0}^{\infty} g(u ; \alpha-\theta) \int_{0}^{\infty} g(v ; \beta+\theta) \Phi^{c}\left(\frac{\gamma-u+v-\mu \rho T-\theta \sigma^{2} \rho T}{\sigma \sqrt{\rho T}}\right) d v d u .
$$

For given parameter values the double integral (25) can be evaluated numerically quite quickly and thence via (24) and (23) the option value computed.

Fig. 4 shows the difference (vertical axis) between the Black-Scholes option value (assuming a normal distribution for logarithmic daily returns) and the option value assuming a GNL distribution for various values of the current stock price (horizontal axis). The strike price was set at $K=1$ and the discount rate at $r=0.05$ per annum. The distribution of daily logarthmic returns was assumed to be $\operatorname{GNL}\left(\mu=0, \sigma^{2}=0.02, \alpha=17.5, \beta=17.5, \rho=0.1\right)$. This has mean zero and variance of 0.00165 , which was used in computing the Black-Scholes option value. The coefficient of kurtosis is 4.68 , which is close to the value of 4.73 observed for a sequence of 929 logarithmic returns for IBM common stock over the period Jan, 1999 - Sept., 2003. The three curves correspond to exercise dates $T=10,30$ and 60 days in advance.

It can be seen in Fig. 4 that "at the money" $(S=1)$ the Black-Scholes price is too high. Although the difference is less than one tenth of one cent it amounts to about 1.5 percent (for $T=10$ ) of the Black-Scholes option value. The corresponding percentages for $T=30$ and $T=60$ are about 0.5 percent and about 0.3 percent. The reason why the difference decreases as $T$ increases is that distribution of log-returns $\left(\operatorname{GNL}\left(\mu, \sigma^{2}, \alpha, \beta, \rho T\right)\right)$ is closer to normality for larger $T$ (a central limit effect).

Far enough "in the money" $(S>1)$ or "out of the money" $(S<1)$ the 
Black-Scholes valuation is too low. This is because the normal model fails to anticipate more extreme fluctuations, which are slightly more likely to occur with the GNL distributed daily returns.

\section{Estimation for NL and GNL distributions.}

For the NL distribution maximum likelihood estimation of parameters can be carried out numerically since there is a closed-form expression for the pdf. In fact it is shown in Reed \& Jorgensen, (2003) how one can estimate $\mu$ analytically and then maximize numerically the concentrated (profile) log-likelihood over the remaining three parameters. Another approach, also discussed by Reed \& Jorgensen, uses the EM-algorithm (considering an NL random variable as the sum of normal and Laplace components, with one regarded as missing data).

Things are more difficult for the GNL distribution, since there is no apparent closed-form expression for the pdf. It may be possible to use the EM-algorithm, but calculating the conditional expectations required appears to be a formidable task. Parameter estimates can be obtained by the method of moments (solving the equations produced by setting the first five sample cumulants equal to their theoretical counterparts, using (19)). This can be achieved by solving numerically a pair of equations (in $\alpha$ and $\beta$ ) and then obtaining the solutions for the other parameters by substitution. One drawback with the method of moments is that it is difficult to impose constraints on parameters (such as requiring estimates of $\alpha, \beta, \rho$ and $\sigma^{2}$ be positive) and estimates which are unsatisfactory in this respect may sometimes occur. 


\section{REFERENCES}

Barndorff-Nielsen, O. E., 1977, Exponentially decreasing distributions for the logarithm of particle size. Proc. R. Soc. Lond. A., 353:401-419

Barndorff-Nielsen, O. E., 1997. Normal inverse Gaussian distributions and stochastic volatility models Scan. J. Stat., 24:1-13.

Eberlein E. and U. Keller, 1995. Hyperbolic distributions in finance. Bernoulli 1:281-289.

Kotz, S., T. J. Kozubowski and K. Podgórski, 2001. The Laplace Distribution and Generalizations. Birkhäuser, Boston.

Reed, W. J. and M. Jorgensen, 2004. The double Pareto-lognormal distribution - A new parametric model for size distributions. Com. Stats - Theory \& Methods, 33:1733-1753.

Rydberg, T. H., 2000. Realistic statistical modelling of financial data. Inter. Stat. Rev., 68, 233-258.

Schoutens, W., 2003. Lévy Processes in Finance, J. Wiley and Sons, Chichester.

Wilson, E. B., 1923. First and second laws of error. J. Amer. Stat. Assoc. 18:841-852. 


\section{Figure Captions.}

Figure 1 Solid curve - the normal-Laplace density with $\mu=0, \sigma^{2}=1 / 3, \alpha=$ $1 / \sqrt{3}, \beta=1 / \sqrt{3}$ which has mean 0 and variance 1 ; dot-dash curve - standard normal density; and dashed curve - the Laplace density with mean zero and variance 1.

Figure 2 The density of the $\mathrm{NL}(0,1,1, \beta)$ for (moving down the peaks) $\beta=$ $1,1 / 2,1 / 3,1 / 4$ and $1 / 5$.

Figure 3 The density of the symmetric normal-Laplace distribution $(\mathrm{NL}(0,1, \alpha, \alpha))$ for (moving down the peaks) $\alpha=2,1,3 / 4$ and $1 / 2$.

Figure 4 The difference between option values for a European call option using a normal distribution (Black-Scholes option value) and a generalized normalLaplace (GNL) distribution for the $\log$ (price) increments. The horizontal axis shows the current stock price, $S$, and the vertical axis the difference in option values. The strike price was set at $K=1$; the per-annum discount rate at $r=$ 0.05 ; the GNL parameter values at $\mu=0, \sigma^{2}=0.02, \alpha=17.5, \beta=17.5, \rho=0.1$; and the normal distribution for computing the Black-Scholes option value had mean 0 and variance 0.00165 , the same as those of the GNL. The three curves correspond to excercise times (moving down the peaks) $T=10,30$ and 60 days ahead. 

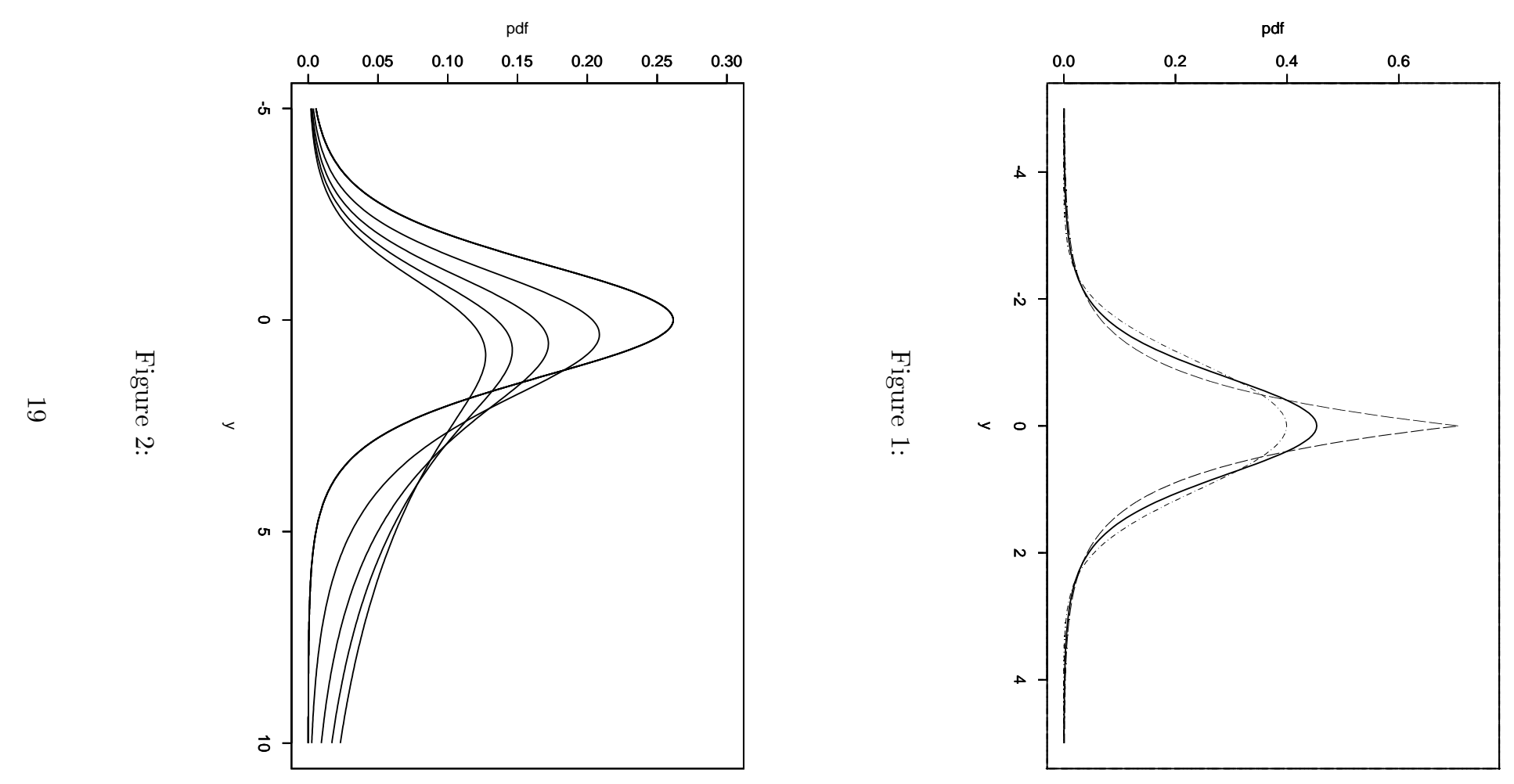

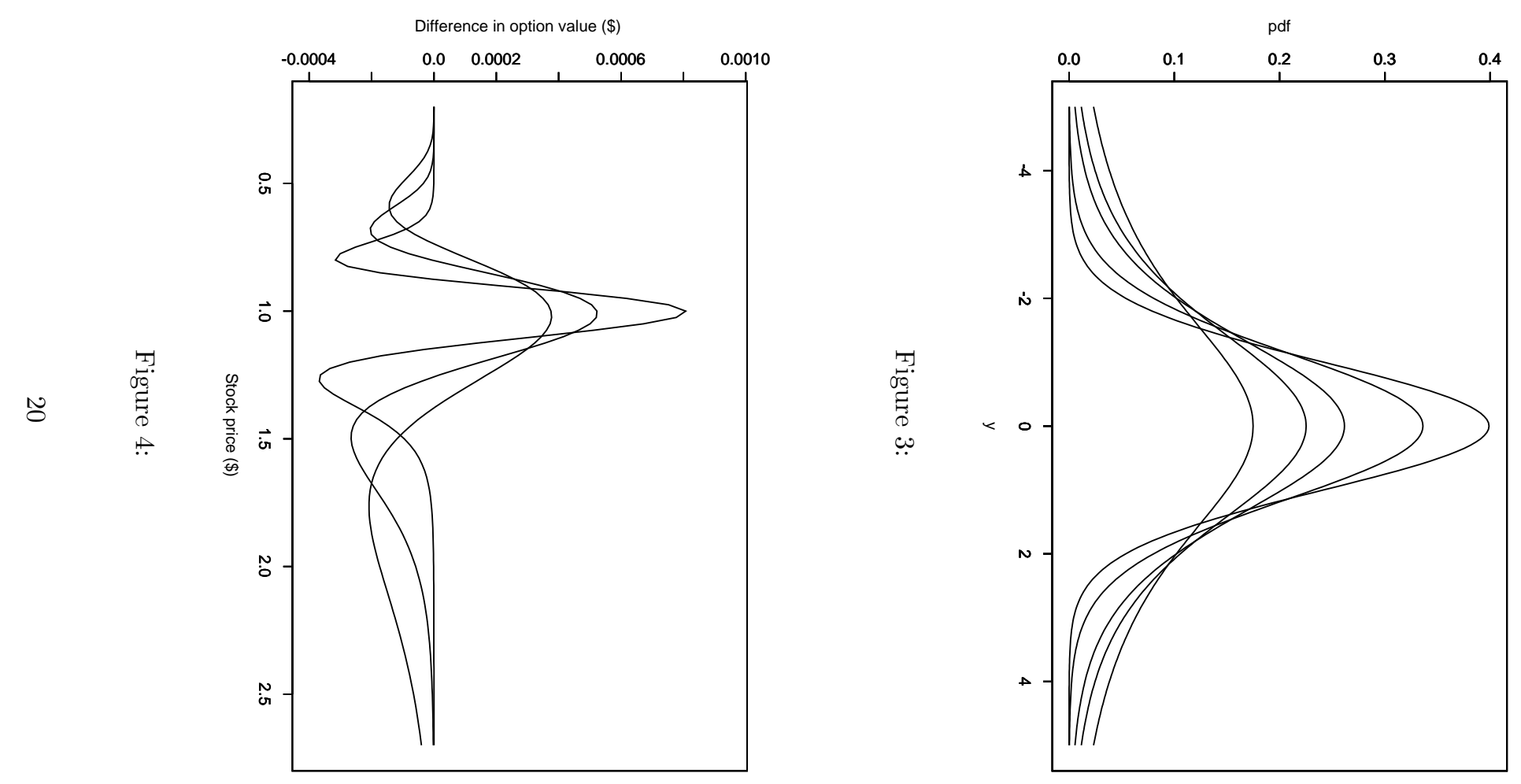